\title{
Validation of analysis results using diode array detection combined with a chromatography data system
}

\author{
L. Jaufmann \\ Kontron Instruments SpA, Via Fantoli 16/15, 20138 Milan, Italy
}

\begin{abstract}
Careful method validation, demonstrating that the analytical method is suitable for the intended use, and frequent system suitability tests are the basis for high quality analysis results. Diode array detection, providing high resolution spectra at any time, adds further quality information about component identity and peak purity. Stateof-the-art diode array detectors demonstrate sensitivity comparable to the best conventional variable wavelength detectors alongside high resolution spectra.
\end{abstract}

For identification and quantitative tests for impurity content, the use of diode array detectors or mass detectors is recommended.

\section{System suitability test}

Frequent system suitability testing is required to demonstrate that the analysis system is still performing within the limits of the original method validation. A modern chromatography system supports the analyst in these routine tests by executing the check automatically, avoiding any extra effort and possible human errors.

Acceptance limits, verified by the analytical method validation, are entered into an application specific limit table (Fig. 1). In addition, the system behaviour in case of a limit violation is pre-defined in the method. Specific control samples for the system suitability test are inserted into the sample sequence. Control charts are useful tools to visualise long-term system performance and to identify trends requesting corrective actions before analysis results become invalid.

\section{Why use a diode array detector?}

- identification tests

- quantitative tests for impurities' control

- limit tests for the control of impurities

- quantitative tests on the active component

for the following validation characteristics:

- accuracy

- precision (repeatability and intermediate precision)

- specificity

- detection and quantitation limits

- linearity

- range.

\section{Method validation}

Method validation is proving that an analytical method is suitable for the intended purpose. Validation is requested aECD publications and ISO guidelin Common rules for analytical method validation, described in the ICH guidelines, are meanwhile accepted by all authorities.

The ICH guidelines require validation on: 


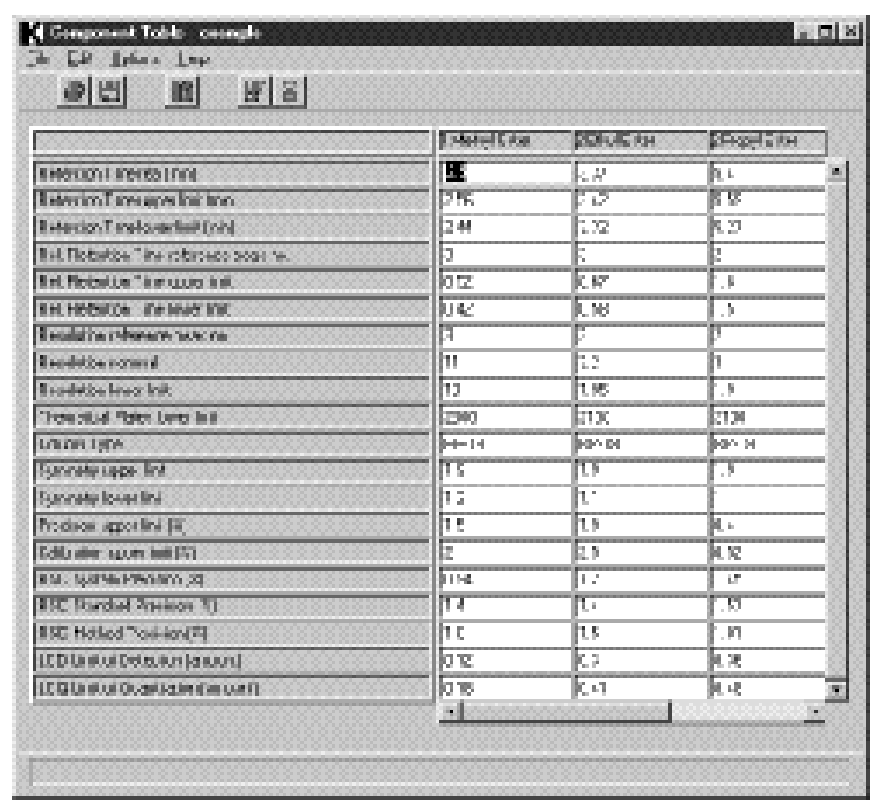

Figure 1. Limit table for system suitability test.

\section{Component identity}

Component identity should be checked by spectra library comparison using high resolution spectra. High spectral resolution is required to indicate the difference on similar molecular structures. Impurities and by-products, generated during a synthesis process, are typically very similar to the active component itself, and therefore the spectra show only minor differences. Only diode array detectors with an extremely high spectral resolution, typically better than $1 \mathrm{~nm}$, permit differentiation between such spectra. Lower spectral resolution from the diode array detector can lead to wrong component identification or a too high matching factor during the spectra library search.

Many diode array detectors currently in use feature only poor spectral resolution. In such cases even significantly different spectra are displayed similarly, the real difference is not clearly visible. Figure 2 shows the comparison between Benzene and Anthracene spectra at $5 \mathrm{~nm}$ resolution.

High resolution diode array detectors such as the Kontron Instruments Diode Array Detector 540 provide maximum spectral resolution of $0.8 \mathrm{~nm}$ per diode; a resolution comparable with a high performance spectrophotometer. Spectra comparison between Benzene and Anthracene spectra are now demonstrating the major difference between the two components, both graphically (Fig. 3) and in match factor calculation.

\section{Peak purity}

Peak purity testing in routine analysis adds important quality information to analysis results. A conventional single channel variable wavelength detector offers only quantitative information; information on peak purity is completely miss-

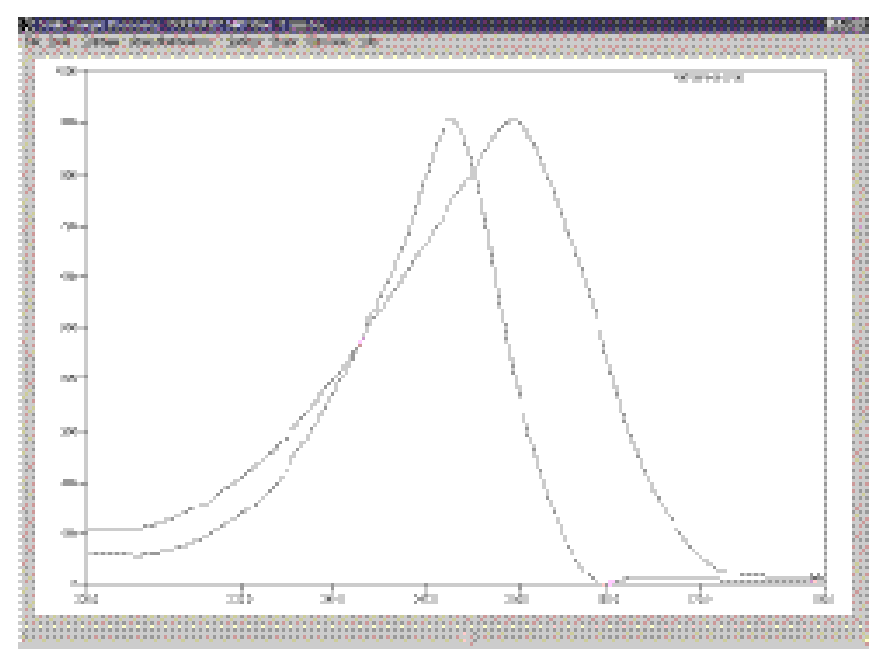

Figure 2. Comparison Benzene - Anthracene at $5 \mathrm{~nm}$ resolution.

ing. Conventional multi-wavelengths detectors allow to calculate the ratio between two wavelength signals as a first purity indication. If the spectral difference between the main component and the chemically similar impurity is only visible at a spectral range different from the two selected wavelengths, the impurity becomes invisible for this detection technique.

The only reliable information on peak purity is obtained by comparing all spectra under a peak. High resolution spectra comparison is mandatory because impurities from similar molecule structures, e.g. degradation products, generated during the production process, must be identified. When comparing low resolution spectra, impurities may become invisible and the purity factor is calculated as being too high.

The combination of a high performance diode array detector and a chromatography data handling system such as the Kontron Instruments DAD 540 and KromaSystem 2000 significantly improves the quality of analysis results by

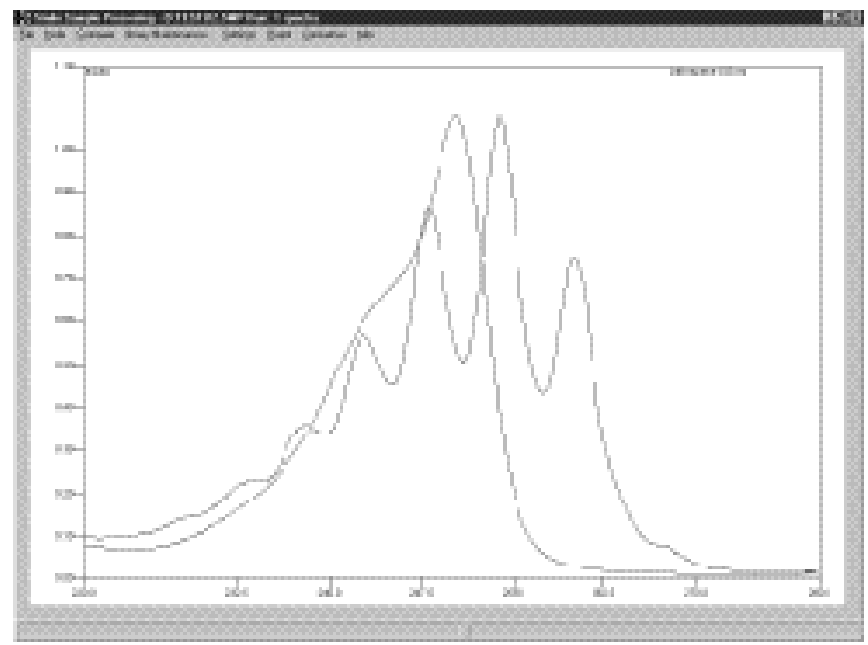

Figure 3. Comparison Benzene - Anthracene at $0.8 \mathrm{~nm}$ resolution. 
automatically checking peak purity, based on comparison of all high resolution spectra under a peak. Peak purity is indicated graphically and in the spectra report (Fig. 4). The spectra report includes the spectra library search result as well as the purity factor.

Because $3 \mathrm{~d}$ data require a huge amount of memory space, KromaSystem 2000 allows to pre-select acceptance limits for component identity and peak purity. In case component identity and peak purity are verified as required, the $3 \mathrm{~d}$ data are printed as contour plot or as a real $3 \mathrm{~d}$ image, relevant spectra are automatically extracted and then the huge $3 \mathrm{~d}$ data field is automatically deleted. When a limit violation is detected, the $3 \mathrm{~d}$ data field remains stored for further investigation by the user. This automatic limits check allows therefore use of high resolution diode array detection even for routine analysis, adding the maximum information on component identity and peak purity.

\section{Why compromise sensitivity and resolution?}

Most diode array detectors in use compromise between chromatogram sensitivity and spectral resolution. A state-of-theart diode array detector such as the Kontron Instruments DAD 540 features both simultaneously in one run. The polychromator design based on a ceramic optical bench is the basis for extremely high sensitivity. A noise specification below $10 \mu \mathrm{Abs}$, combined with an unmatched drift in diode array technology, guarantees chromatograms for quantitative calculations similar to the best conventional detectors (Fig. 5). These extremely sensitive quantitative analysis results are further enhanced by perfect identity and purity information.

\section{Conclusion}

A conventional chromatography system, using a variable wavelength detector and an integrator, delivers only quantitative information, while qualitative information is entirely missing.

The quality of analysis results is significantly improved by diode array detection in combination with a chromatog- raphy data handling system by automatically checking the system suitability and indicating component identity and peak purity based on high resolution spectra.

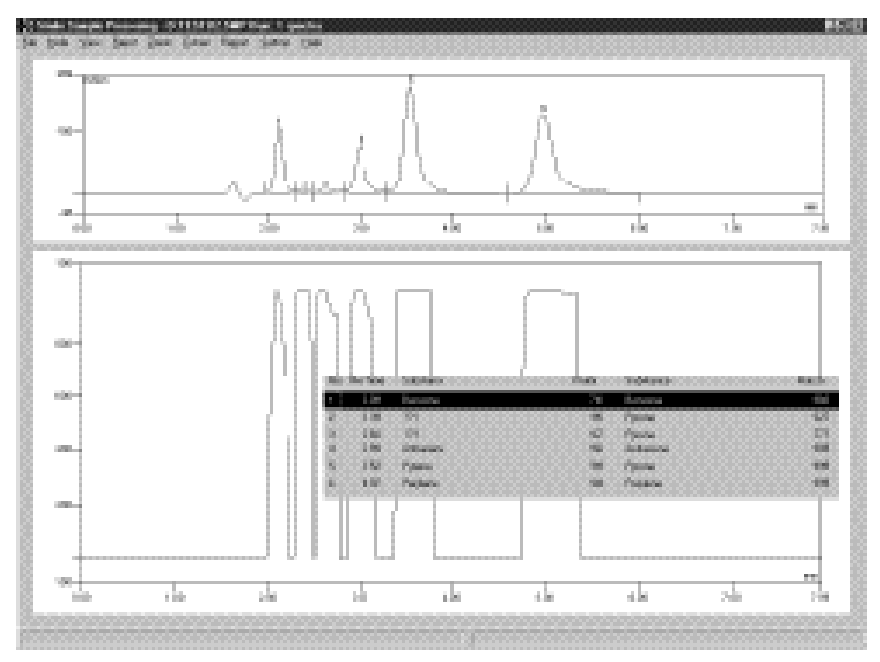

Figure 4. Peak purity.

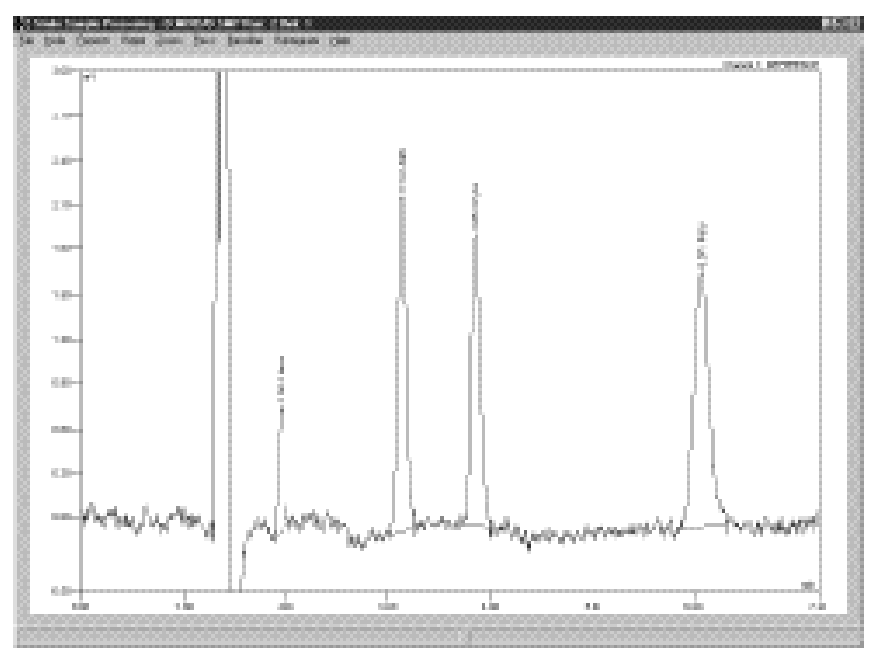

Figure 5. High sensitive chromatogram. 